\title{
Pediatric Pulmonology Year in Review 2020: Physiology
}

Angela Delecaris ${ }^{1}$, Samantha Averill, ${ }^{1}$, Jonathan Krasinkiewicz ${ }^{1}$, Jessica Saunders ${ }^{1}$, and Clement Ren ${ }^{2}$

${ }^{1}$ Indiana University School of Medicine

${ }^{2}$ Indiana University System

April 4, 2021

\begin{abstract}
Pulmonary physiology is a core element of pediatric pulmonology care and research. This article reviews some of the notable publications in physiology that were published in Pediatric Pulmonology in 2020.
\end{abstract}

Pediatric Pulmonology Year in Review 2020: Physiology

Angela O. Delecaris MD

Samantha H. Averill MD

Jonathan Krasinkiewicz MB BCh BAO

Jessica L Saunders MD

Clement L. Ren MD MBA

${ }^{1}$ Division of Pediatric Pulmonology, Allergy, and Sleep Medicine, Riley Hospital for Children, Indianapolis, IN. USA

${ }^{2}$ Department of Pediatrics, Indiana University School of Medicine, Indianapolis, IN USA

Corresponding author:

Clement L. Ren, MD, MBA Riley Hospital for Children ROC 4270705 Riley Hospital Drive Indianapolis, IN 46202 Phone: 317-948-7180 FAX: 317-944-7247 Email: clren@iu.edu

Conflicts of Interests:

AOD has no conflicts of interest to disclose. SA has no conflicts of interest to disclose. JK has no conflicts of interest to disclose. JBS has no conflicts of interest to disclose. CLR has no conflicts of interest to disclose. Running Title:

Pediatric Pulmonology Year in Review 2020: Physiology

Keywords:

Multiple breath washout, Tidal breathing, Pulmonary function testing, Forced oscillometry, Bronchopulmonary dysplasia

Abstract 
Pulmonary physiology is a core element of pediatric pulmonology care and research. This article reviews some of the notable publications in physiology that were published in Pediatric Pulmonology in 2020.

\section{Introduction}

Physiology is one of the foundations of pediatric pulmonology, and in 2020 there were several important articles focused on pediatric pulmonary physiology published in Pediatric Pulmonology .

\section{Forced Oscillometry}

Forced oscillometry (FO) uses measurement of pressure and flow in response to an oscillatory pressure wave introduced at the airway opening to measure respiratory system impedance (Z) [1]. Z is a generalized concept of resistance that incorporates the in phase and out of phase relationships between pressure and flow. The in phase element of $\mathrm{Z}$ is resistance $(\mathrm{R})$, while the out of phase element is represented by reactance (X). $\mathrm{R}$ is composed of the forces associated with frictional losses in the airways and the lung parenchyma. $\mathrm{X}$ is composed of an inertive element $(\mathrm{Xi})$, which represents the inertive forces of the respiratory system, and a capacitant element $(\mathrm{Xc})$, which reflects the visco-elastic properties of the lung. At low frequencies, Xc predominates over Xi. Because FO does not require a maximal forced expiratory maneuver, it is easier to obtain measurements from young children compared to spirometry. $\mathrm{X}$ and $\mathrm{R}$ at low frequency may also reflect small airways function better than spirometric measures such as the forced expiratory volume in 1 second (FEV1).

There is increasing evidence that the lungs are affected by diabetic microangiopathy. Anik, et al performed FO and spirometry on children with type 1 diabetes mellitus (T1DM) and compared the results to data from age matched healthy controls [2]. $\mathrm{R}$ at 5, 10, and $20 \mathrm{~Hz}$ (R5, R10, and R20 respectively) was significantly higher in children with T1DM compared to controls, and the forced expiratory flow at $75 \%$ of forced vital capacity $\left(\mathrm{FEF}_{75}\right)$ and between $25-75 \%$ of forced vital capacity $\left(\mathrm{FEF}_{25-75}\right)$ were both significantly lower. Lung function was worse in children with poorly controlled T1DM compared to well-controlled T1DM. These results demonstrate subclinical impairment of lung function in children with T1DM and suggest that monitoring of lung function in T1DM should be a part of T1DM care.

Zheng, et al studied whether impulse oscillometry (IOS), a form of FO, and the fraction of exhaled nitric oxide (FeNO) could be used to predict future asthma exacerbations in preschool children [3]. They were able to identify a cutoff value for the area under the reactance curve (AX), a measure of total low frequency X, that predicted future loss of asthma control, while FeNO did not have strong predictive value in this patient population. Their report suggests that IOS could be used to monitor preschool children for future risk of asthma exacerbation.

Lundberg, et al investigated the feasibility and correlation between spirometry and IOS in 6 year old children born extremely premature [4]. The success rate of IOS was significantly higher in both term and preterm children compared to spirometry (93\% vs 60\%). There was moderate correlation between spirometry measurements and IOS measurement, suggesting that IOS could be considered as an alternative test in children who cannot perform spirometry.

Veneroni, et al used FO to compare respiratory mechanics in preterm infants treated with continuous positive airway pressure (CPAP) versus $\mathrm{CPAP}$ with sustained inflations of $25 \mathrm{cmH} 2 \mathrm{O}$ for 15 seconds [5]. Infants presented with highly heterogeneous degrees of lung aeration at birth, limiting their ability to use FO to assess ventilation strategies. However, this pilot study did demonstrate the feasibility of obtaining FO measurements at birth in preterm infants.

\section{Multiple Breath Washout}

The multiple breath washout (MBW) technique can be used to assess ventilation inhomogeneity, which can be seen in a variety of pediatric lung diseases, most notably cystic fibrosis (CF) [6]. MBW can be performed either by washing in a tracer gas, such as sulfur hexafluoride, and then washing the tracer gas out with 
ambient air or washing out the resident nitrogen $\left(\mathrm{N}_{2}\right)$ in the lungs with $100 \%$ oxygen. The lung clearance index (LCI) is the most commonly used measure derived from $\mathrm{N}_{2} \mathrm{MBW}$, and it represents the number of lung turnovers required to washout resident $\mathrm{N}_{2}$ from the lungs down to $2.5 \%$ of the original concentration; a higher LCI indicates increased ventilation inhomogeneity. In addition to LCI, other measures derived from MBW include $\mathrm{LCI}_{5.0}$, where $\mathrm{N}_{2}$ is washed out to $5 \%$ of the original concentration, slope of phase III indices, and moment ratio analysis.

Parisi, et al performed MBW and spirometry in a group of childhood cancer survivors and matched healthy controls [7]. They found that LCI was higher in these children compared to healthy controls, but this difference was not statistically significant; and there was no difference in spirometry measurements. There was a significant correlation between years following chemotherapy and increased LCI. Their results suggest that the chemotherapy used to treat childhood tumors may have less pulmonary toxicity than previously expected, at least during childhood. The increase in LCI over time suggests that LCI could be used to non-invasively assess pulmonary fibrosis but larger prospective studies are needed.

Gambazza, et al evaluated the association of LCI with exercise capacity in patients with CF [8]. LCI was significantly higher in patients with exercise intolerance, but it was also higher in some patients with normal exercise tolerance and normal to moderate airflow obstruction. No association was found between predicted peak work and LCI, but there was a significant association between peak work and nutritional status, airflow obstruction, and chronic Pseudomonas aeruginosainfection. Based on these findings, providers should not assume that patients with normal exercise tolerance do not have early lung disease.

Irving, et al compared MBW results between children with severe treatment resistant asthma (STRA), difficult asthma (DA) and healthy controls [9]. LCI was significantly higher in the patients with STRA compared to those with DA and controls but there was no difference between DA and controls. FeNO and LCI showed significant improvement following parenteral steroids. These results suggest that distal airways disease is worse in patients with STRA and can sometimes be improved with parenteral steroids. The improvement in FeNO and LCI suggests these patients with STRA may have distal airway eosinophilic inflammation that may not respond to inhaled corticosteroids. These results suggest that FeNO and LCI may be useful in monitoring treatment response in patients with STRA.

Other studies of MBW focused on use of outcome measures other than LCI and comparison of difference MBW devices. Arigliani, et al compared the conduction-dependent contribution to ventilation inhomogeneity $\left(\mathrm{S}_{\text {cond }}\right)$, the diffusion-convection interaction-dependent inhomogeneity $\left(\mathrm{S}_{\mathrm{acin}}\right)$, LCI, spirometry, and transfer capacity of the lung to carbon monoxide (TLCO) in children born at $<28$ weeks gestational age (GA) [10].

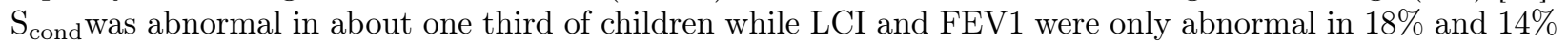
respectively. They did not identify any significant difference in $\mathrm{S}_{\text {comd }}$ between infants born prematurely with BPD compared to those without BPD. No significant difference was observed in $\mathrm{S}_{\text {acin }}$ between the premature infants and the control group and no significant difference was found when comparing those with BPD to those without BPD. Finally, extremely preterm children had lower TLCO compared to controls,Although moment ratios correlated with pulmonary symptoms, high variability limits its utility in the clinical setting. These results suggest that $\mathrm{LCI}_{5.0}$ can be used to track disease progression in young children with $\mathrm{CF}$. Compartment analysis (CA) allows partitioning of MBW data into slower and faster ventilating units. Skov, et al applied $\mathrm{CA}$ to MBW testing in children with CF [12]. Because CA requires an extremely regular breathing pattern, it could only be successfully performed in $53 \%$ of children with CF. However, CA did correlate with other MBW measurements, such as LCI. Further research is needed to determine the clinical value of CA. Isaac, et al used a lung model to compare functional residual capacity (FRC) and LCI measured with 2 different MBW devices and body plethysmography [13]. Not only were there differences in these measurements using these 3 different methods, but for one device LCI decreased with age. These results indicate that MBW data obtained on one device cannot be directly compared to data from other devices.

\section{Reference Equations}

Normal reference values are critically important for interpreting the results of pulmonary function tests 
(PFTs), but data in non-White populations are lacking. Several reports in PediatricPulmonology this past year sought to fill this knowledge gap. Al Querem et al. evaluated the applicability of the 2012 Global Lungs Initiative (GLI) reference equations in 582 Jordanian children ages 6-13 year-old [14]. The GLI equation for Caucasians demonstrated a reasonable fit to the data collected in this study and was more suitable than the GLI equation for other ethnic groups, and equations derived from other studies conducted in Arabic counties. These results differ from previously reported values in Jordanian adults which showed that the GLI equations for other ethnicities rather than GLI Caucasian equation were better correlated, and demonstrate the importance of identifying age-specific reference equations.

Reference values for infant PFT's have been reported but there is a lack of information about racial or ethnic differences. The aim of a study by Kosma et al was to estimate the extent to which previously published reference equations for infant spirometry could be implemented in a cohort of Swedish term, healthy infants [15]. Tidal volume and raised volume rapid thoracoabdominal compression (RVRTC) maneuvers were performed on 91 term infants. Height and weight in Swedish infants were above average compared to other infant PFT reference cohorts, and this led to an underestimation of lung function measured by infant spirometry in the youngest age group and an overestimation in the older infants using previously published infant PFT reference data.

Not only spirometry reference values but also reference equations to calculate diffusing capacity of carbon monoxide (DLCO) have been tested for their applicability in different racial groups. A study by Dridi et al. measured nitric oxide/carbon monoxide transfer in 118 healthy Tunisian males ages 8-14 [16]. They confirmed previous observations that height and body weight are key individual predictors of DLCO and that alveolar volume (VA) was related with both height and weight. Their values for DLCO and VA were similar to those found by Kim et al in both Caucasian and African American populations [17].

Normal reference data for maximal voluntary ventilation (MVV) in children have only been reported for limited ethnic and racial populations. Silva et al. obtained MVV data from children in São Paolo, Brazil [18]. MVV in their cohort differed from results obtained in other populations, highlighting the need and importance to obtain normal reference data for multiple different populations.

Although not the focus of studies published in PediatricPulmonology in 2020, the issue of incorporating race into PFT reference equations has grown in prominence and importance in the past few years. Although genetic ancestry contributes to differences in PFT values in different populations, race is a complex construct that incorporates more than just genetic ancestry, and includes cultural and social factors [19]. Furthermore, race serves as a proxy for social determinants of health that may affect lung function. It is likely that in the future, we will see studies in PediatricPulmonology that more directly incorporate genetic ancestry into predictive equations rather than phenotypic race [20].

\section{Tidal Breathing Studies}

Tidal breathing analysis (TBA) is an attractive method of respiratory function testing because it can be done without the need for sedation or the requirement of forced expiratory maneuvers [21]. TBA can be performed using a face mask and pneumotachometer, but this can be challenging to apply in infants since it requires the infant to maintain a seal over the nose and mouth with the mask, which can also potentially alter infant breathing patterns. Electromagnetic inductance plethysmography (EIP) measures changes in the electromagnetic field created by tidal breathing while wearing a metallic vest through which a low voltage is applied. Sautter, et al used EIP to perform TBA in a cohort of 36 infants with bronchiolitis [22]. EIP was successfully performed in $80 \%$ of the infants, and a significant increase in the ratio of the total expiratory time over the inspiratory time $(\mathrm{Te} / \mathrm{Ti})$ was observed after inhalation of normal saline. The ratio of the time to peak expiratory flow over total expiratory time (Tptef/Te) decreased after administration of nebulized normal saline, but this change was not statistically significant. This study demonstrates the feasibility of using EIP for TBA and suggests that further study of the physiologic effects of inhaled normal saline in bronchiolitis are warranted. Traditionally TBA has been done manually, which is a time consuming and laborious process. A study by Gomes et al. compared two computing systems, AutoCAD and MATLAB, 
in their ability to obtain automated TBA data [23]. Thoracoabdominal area averages calculated with both systems were comparable, but MATLAB required less time and less user training to complete. They suggest that it could be used in the clinic setting to calculate or derive tidal volume measurements.

\section{Physiologic Outcomes of Prematurity}

Bronchopulmonary dysplasia (BPD) is the most common short-term complication of premature birth, and infants with BPD can continue to have impaired lung function into adulthood [24]. Although there have been studies comparing lung function between infants with BPD and those without this diagnosis, there are limited data in preterm infants who are born small for gestational age (SGA). Kim, et al investigated the differences in airway obstruction in term, healthy neonates and preterm neonates with and without BPD using MBW and TBA [25]. No significant differences in Tpef/Te were observed between the term infants, preterm infants with mild/moderate $\mathrm{BPD}$, and preterm infants with severe BPD. However, Tpef/Te was significantly lower in the severe BPD infants compared to the mild/moderate BPD infants, consistent with worse airway obstruction in the former group. LCI was significantly increased in preterm infants compared to term ones, but it did not vary between the infants with no BPD, mild BPD, or severe BPD, even when accounting for SGA status. SGA infants with BPD had a significantly lower Tpef/Te than average for GA matched infants, showing a negative effect of SGA in BPD. These results suggest that SGA status is a risk factor for worse airway obstruction in infants with BPD.

Sex differences in lung function and structure are well known, but studies of sex differences in lung mechanics of preterm neonates are lacking. Kim, et al measured airway pressure and flow in male and female neonates requiring mechanical ventilation for neonatal respiratory distress syndrome, and then used these data to generate a single compartment model to calculate specific elastance $\left(\mathrm{E}_{\mathrm{S}}\right)$ [26]. Male infants had higher $\mathrm{E}_{\mathrm{S}}$ compared to female infants of matched gestational age and less breath-to-breath variability in $\mathrm{E}_{\mathrm{S}}$. This study suggests that ventilator strategies may need to take sex into account, but further studies with larger cohorts are needed.

0.5. There was no significant difference in any other forced expiratory flow measurements, FRC, or respiratory system compliance between the caffeine treated or untreated groups. When further stratified by BPD diagnosis, differences in lung function were only seen in infants without BPD who had received caffeine. These results indicate that caffeine may have beneficial effects on lung function in addition to its effect on reducing risk of $\mathrm{BPD}$.

\section{Other Physiologic Studies}

A study by Fabi et al. examined short- and long-term changes in respiratory mechanics after palliative or corrective surgery in 168 infants and toddlers with congenital heart disease [28]. They found that these patients demonstrated normal basal respiratory function but decreased exercise capacity. This decrease in exercise capacity was more pronounced in patients who underwent total cavopulmonary connection (TCPC) procedures. This suggests the need to conduct respiratory monitoring on patients with TCPC more closely and for longer than those with non-TCPC procedures. They also found that a higher age at surgery but prior to 24 months was associated with better respiratory outcomes which suggests that increased development of the lung parenchyma prior to intervention will lead to improved respiratory outcomes post-operatively.

Sivieri, et al used a preterm infant lung model to evaluate the effects on end tidal $\mathrm{CO}_{2}$ (ETCO2) when high frequency oscillation is superimposed on bubble CPAP (BCPAP) [29]. They found that there is improved $\mathrm{CO}_{2}$ clearance when high frequency oscillation is applied to BCPAP, and that this effect was more pronounced when the model mimicked lung disease in a preterm infant compared to normal infant lung mechanics. This improvement was not dependent on BCPAP pressure, CO2 clearance was significantly improved for both lung models as a function of increased frequency and lower respiratory rate. These results support conducting a clinical trial to assess the effect of superimposed high frequency oscillations in infants receiving BCPAP.

Extrathoracic high frequency chest compression (HFCC) using an inflatable vest is frequently used for airway clearance in patients with mucociliary abnormalities, such as CF. Weiner, et al sought to determine 
if HFCC augments ventilation by measuring end-tidal CO2 (ETCO2) after HFCC was performed at varying frequencies $(\mathrm{Hz})$ in a cohort of 15 people with $\mathrm{CF}$ who were experiencing a pulmonary exacerbation [30]. ETCO2 values were significantly lower at all $\mathrm{Hz}$ compared to no treatment. This remained the same when adjusting for patient specific factors such as age, sex, body mass index, baseline FEV1 and respiratory rate. Increasing $\mathrm{Hz}$ produced a small, but significant improvement in ETC02 clearance. These results demonstrate that HFCC increases clearance of $\mathrm{CO} 2$ in a frequency dependent manner. Further research is needed to determine the clinical importance of this finding.

Formulas used to calculate the appropriate pediatric tracheostomy or endotracheal tube (ETT) size have incorporated age, weight, and height, but there are no formulas that incorporate airflow resistance or crosssectional area of the inner cannula. Since resistance varies by the $4^{\text {th }}$ power of the radius, small changes in ETT lumen diameter can have marked effects on tube resistance (R) [31]. Khan and Baker applied the Hagen-Poiseuille equation for $\mathrm{R}$ under laminar flow conditions to calculate the $\mathrm{R}$ in different ETT and tracheostomy tubes based on tube inner diameter and cross-sectional area [32]. A large decrease in $\mathrm{R}$ was seen when tube diameter was increased by as little as $0.5 \mathrm{~mm}$ in ETTs that would likely be used for patients $<1$ year of age. This effect was not as significant in larger tube sizes. When invasive mechanical ventilation is required, and a patient can safely tolerate upsizing of ETT or tracheostomy tube, this should be considered to lower airway resistance and improve airflow dynamics.

\section{Summary}

Pulmonary physiology continues to play an important role in pediatric pulmonology care and research, and we anticipate that future issues ofPediatric Pulmonology will continue to contain reports of interest and importance on this topic.

\section{References}

1. Goldman MD. Clinical Application of Forced Oscillation.Pulmonary Pharmacology 8 Therapeutics. 2001;14(5):341-50.

2. Anık A, Anık A, and Uysal P. Assessment of pulmonary function by impulse oscillometry and spirometry in children with type 1 diabetes mellitus. Pediatric Pulmonology. 2020;55(12):3517-24.

3. Zheng $\mathrm{S}, \mathrm{Hu} \mathrm{Y}$, Chen Z, Wang M, and Liao W. Predicting asthma exacerbation by impulse oscillometry evaluation of small airway function and fractional exhaled nitric oxide in preschool children.Pediatric Pulmonology. 2020;55(7):1601-7.

4. Lundberg B, Melén E, Thunqvist P, Norman M, and Hallberg J. Agreement between spirometry and impulse oscillometry for lung function assessment in 6-year-old children born extremely preterm and at term.Pediatric Pulmonology. 2020;55(10):2745-53.

5. Veneroni C, Mercadante D, Lavizzari A, Colnaghi M, Mosca F, and Dellacà RL. Changes in respiratory mechanics at birth in preterm infants: A pilot study. Pediatric Pulmonology. 2020;55(7):1640-5.

6. Robinson PD, Goldman MD, and Gustafsson PM. Inert gas washout: theoretical background and clinical utility in respiratory disease.Respiration. 2009;78(3):339-55.

7. Parisi GF, Cannata E, Manti S, Papale M, Meli M, Russo G, et al. Lung clearance index: A new measure of late lung complications of cancer therapy in children. Pediatric Pulmonology. 2020;55(12):3450-6.

8. Gambazza S, Guarise R, Carta F, Ambrogi F, Mirabella M, Brivio A, et al. Exercise capacity and ventilation inhomogeneity in cystic fibrosis: A cross-sectional study. Pediatric Pulmonology.2020;55(2):394-400.

9. Irving S, Fleming L, Ahmad F, Biggart E, Bingham Y, Cook J, et al. Lung clearance index and steroid response in pediatric severe asthma.Pediatric Pulmonology. 2020;55(4):890-8.

10. Arigliani M, Valentini E, Stocco C, De Pieri C, Castriotta L, Barbato V, et al. Regional ventilation inhomogeneity in survivors of extremely preterm birth. Pediatric Pulmonology.2020;55(6):1366-74. 
11. Shaw M, Oppelaar MC, Jensen R, Stanojevic S, Davis SD, Retsch-Bogart G, et al. The utility of moment ratios and abbreviated endpoints of the multiple breath washout test in preschool children with cystic fibrosis.Pediatric Pulmonology. 2020;55(3):649-53.

12. Skov L, Green K, Stanojevic S, Jensen R, Buchvald F, Ratjen F, et al. Lung compartment analysis assessed from N2 multiple-breath washout in children with cystic fibrosis. Pediatric Pulmonology.2020;55(7):1671-80.

13. Isaac SM, Jensen R, Anagnostopoulou P, Davies JC, Gappa M, Latzin P, et al. Evaluation of a multiple breath nitrogen washout system in children. Pediatric Pulmonology. 2020;55(8):2108-14.

14. Al-Qerem WA. How applicable are GLI 2012 equations to a sample of Middle Eastern school-age children? Pediatric Pulmonology.2020;55(4):986-93.

15. Kosma P, Palme-Kilander C, Bottai M, Ljungberg H, and Hallberg J. Forced expiratory flows and volumes in a Swedish cohort of healthy term infants. Pediatric Pulmonology. 2020;55(1):185-9.

16. Dridi R, Dridi N, Ben Moussa Zouita A, Muller PT, Tabka Z, Guénard H, et al. Pulmonary diffusing capacity measured by NO/CO transfer in Tunisian boys. Pediatric Pulmonology. 2020;55(10):2754-61.

17. Kim YJ, Christoph K, Yu Z, Eigen H, and Tepper RS. Pulmonary diffusing capacity in healthy AfricanAmerican and Caucasian children.Pediatr Pulmonol. 2016;51(1):84-8.

18. Silva JC, Carvalho IE, Dal Corso S, and Lanza FC. Reference equation for maximal voluntary ventilation in children and adolescents.Pediatric Pulmonology. 2020;55(2):426-32.

19. Borrell LN, Elhawary JR, Fuentes-Afflick E, Witonsky J, Bhakta N, Wu AHB, et al. Race and Genetic Ancestry in Medicine - A Time for Reckoning with Racism. New England Journal of Medicine.2021;384(5):47480.

20. Kumar R, Seibold MA, Aldrich MC, Williams LK, Reiner AP, Colangelo L, et al. Genetic ancestry in lung-function predictions. $N$ Engl J Med. 2010;363(4):321-30.

21. Palmer J, Allen J, and Mayer O. Tidal Breathing Analysis.NeoReviews. 2004;5(5):e186-e93.

22. Sautter M, Halvorsen T, Engan M, Clemm H, and Bentsen MHL. Electromagnetic inductance plethysmography to study airflow after nebulized saline in bronchiolitis. Pediatric Pulmonology.2020;55(12):3437-42.

23. Gomes DC, Azevedo IG, de Figueiredo Araújo AG, da Costa Lopes LD, Nagem DAP, Magalhães FA, et al. Thoracoabdominal motion in newborns: Reliability between two interactive computing environments.Pediatric Pulmonology. 2020;55(5):1184-9.

24. Jobe AH, and Bancalari E. Bronchopulmonary Dysplasia. Am J Respir Crit Care Med. 2001;163(7):17239 .

25. Kim YH, Kim KW, Eun HS, Shin JE, Sol IS, Kim SY, et al. Small for gestational age birth may increase airflow limitation in bronchopulmonary dysplasia. Pediatric Pulmonology.2020;55(2):346-53.

26. Kim KT, Knopp J, Dixon B, and Chase JG. Mechanically ventilated premature babies have sex differences in specific elastance: A pilot study. Pediatric Pulmonology. 2020;55(1):177-84.

27. Rodríguez Sánchez de la Blanca A, Sánchez Luna M, González Pacheco N, Ramos Navarro C, Santos González M, and Tendillo Cortijo F. New indicators for optimal lung recruitment during high frequency oscillator ventilation. Pediatric Pulmonology. 2020;55(12):3525-31.

28. Fabi M, Balducci A, Cazzato S, Aceti A, Gallucci M, Di Palmo E, et al. Resting respiratory lung volumes are "healthier" than exercise respiratory volumes in different types of palliated or corrected congenital heart disease. Pediatric Pulmonology.2020;55(3):697-705.

29. Sivieri EM, Eichenwald EC, Rub D, and Abbasi S. Carbon dioxide clearance using bubble CPAP with superimposed high-frequency oscillations in a premature infant lung model with abnormal lung mechanics. 
Pediatric Pulmonology. 2020;55(11):3189-96.

30. Weiner GA, Forno E, and Weiner DJ. The effects of high-frequency chest compression on end-tidal CO2. Pediatric Pulmonology.2020;55(3):646-8.

31. West JB, AW Luks. Pulmonary Physiology: The Essentials. Tenth Edition. Wolters Kluwer; 2015.

32. Khan EK, and Baker CD. Differences in cross-sectional area and airflow resistance between pediatric endotracheal or tracheostomy tubes.Pediatric Pulmonology. 2020;55(9):2194-5. 